\title{
A phase II trial of S-I monotherapy in metastatic colorectal cancer after failure of irinotecan- and oxaliplatin-containing regimens
}

\author{
H-C Jeung ${ }^{1,2}$, SY Rha ${ }^{1,2,3}$, BC Cho ${ }^{2,4}$, NC Yoo ${ }^{2,4}$, JK Roh ${ }^{1,2,3,4}$, WJ Roh', HC Chung ${ }^{1,2,3,4}$ and JB Ahn*,2,4 \\ 'Cancer Metastasis Research Center, Yonsei University College of Medicine, Seoul, Korea; ${ }^{2}$ Yonsei Cancer Center, Yonsei University College of Medicine, \\ Seoul, Korea; ${ }^{3}$ BK2 I Project for Medical Science, Yonsei University College of Medicine, Seoul, Korea; ${ }^{4}$ Department of Internal Medicine, Yonsei University \\ College of Medicine, Seoul, Korea
}

This is the first phase II study of S-I monotherapy for patients with metastatic colorectal cancer after failure of both irinotecan- and oxaliplatin-containing regimens. The initial dose of S-I was $35 \mathrm{mg} \mathrm{m}^{-2}$, administered twice daily for 14 days every 3 weeks. Treatment was repeated until the occurrence of disease progression. Twenty-eight patients were enrolled. S- I was administered to 2 I patients as third-line therapy and to the remaining seven patients as fourth-line therapy. Of 26 evaluable patients, the overall response rate was $14.3 \%$ (95\% Cl, 0.4-28. I), and the disease control rate was $42.9 \%$ (95\% Cl, 23.3-62.4). With a median follow-up period of 227 days, median time to progression and overall survival duration were 9 I and $4 \mid 4$ days, respectively. The I-year survival rate of all patients was $60.7 \%$. There was no grade 4 toxicity. Grade 3 haematological toxicities were documented only in two patients. In conclusion, S-I shows potential as a salvage regimen in heavily pretreated colorectal cancer patients. The twice-daily dose of $35 \mathrm{mg} \mathrm{m}^{-2}$ was well tolerated and can be used in designing further combination chemotherapy.

British Journal of Cancer (2006) 95, I637-164I. doi:I0.1038/sj.bjc.6603468 www.bjcancer.com

Published online 14 November 2006

(c) 2006 Cancer Research UK

Keywords: colorectal adenocarcinoma; S- I; oxaliplatin-resistant; irinotecan-resistant

Colorectal cancer is the second leading cause of cancer deaths in Western countries. In Korea, colorectal cancer is the fourth most common malignancy, and its incidence is rising rapidly along with the westernisation of life style (Kim et al, 2002). Thirty percent of patients present with advanced disease, and one-half of patients who have undergone surgery eventually develop metastasis. The prognosis of metastatic disease is poor, although palliative chemotherapy has been shown to prolong survival over best supportive care (Glimelius et al, 1994). 5-Fluorouracil (5-FU) has been widely used in the treatment of metastatic colorectal cancer, and its modulation by leucovorin increased the response rate to $23 \%$.

With the introduction of irinotecan and oxaliplatin during the last decade, treatment choice for metastatic colorectal cancer has been extended. Irinotecan or oxaliplatin combined with 5-FU resulted in improved tumour response with a moderate survival prolongation (De Gramont et al, 2000; Douillard et al, 2000; Saltz et al, 2000). Moreover, there have been recent trials introducing these agents in adjuvant therapy (Alberts et al, 2005; Tyagi et al, 2005). This rapid change in treatment strategy will ultimately lead to a considerable increase in the number of patients with recurrent or progressive disease who have been previously exposed to irinotecan and oxaliplatin, and it raises the necessity for salvage

\footnotetext{
* Correspondence: Professor JB Ahn, Yonsei Cancer Center, Yonsei University College of Medicine, 134, Shinchon-Dong, Seodaemun-Ku, CPO Box \#Seoul 120-752, Korea; E-mail: vvswm5 I3@yumc.yonsei.ac.kr Received 7 June 2006; revised I 4 August 2006; accepted II October 2006; published online 14 November 2006
}

therapy for this patient group. Currently, chemotherapeutic option for these patients is quite limited. Cetuximab is regarded to be a promising option in this setting, and capecitabine is under study of alternative dosing method for use in combination regimens, and investigational agents such as bevacizumab have been tried restrictively for selected patients (Cunningham et al, 2004; Saltz et al, 2004; Gubanski et al, 2005; Matin et al, 2005).

Oral chemotherapy has advantages in the aspects of pharmacoeconomics and patient preference. Oral fluoropyrimidines are considered to be an alternative to conventional protracted 5-FU infusion as far as they provide comparable efficacy and compliance. A novel oral fluoropyrimidine, S-1, has been developed to improve the therapeutic index of tegafur (FT), and 5-FU level was maintained high in plasma and tumour with less gastrointestinal toxicity by combination with two biomodulators, 5-chloro-2,4-dihydroxypyridine (CDHP) and potassium oxonate (Oxo). S-1 has shown promising activity in untreated colorectal cancer through several phase II trials. And in a phase I study, S-1 led to objective response in heavily pretreated colorectal cancer patients (Chu et al, 2004). Based on these results, we conducted a phase II study to evaluate the possibility of S-1 as a salvage option for heavily pretreated colorectal cancer patients who had previously received irinotecan and oxaliplatin.

\section{PATIENTS AND METHODS}

\section{Patient eligibility}

The eligibility criteria were as follows: histologically proven colorectal adenocarcinoma with metastatic, inoperable disease, Eastern 
Cooperative Oncology Group (ECOG) performance scale $0-2$, documented disease progression during or within 6 months after treatment with irinotecan- and oxaliplatin-containing regimens, at least one unidimensionally measurable lesion as assessed by spiral computed tomography outside any previously irradiated area, age $\geqslant 18$ years old, life expectancy $\geqslant 3$ months, and adequate organ functions (WBC $\geqslant 3000 \mu \mathrm{l}^{-1}$, platelets $\geqslant 100000 \mu \mathrm{l}^{-1}$, haemoglobin $\geqslant 9.0 \mathrm{~g} \mathrm{dl}^{-1}$, serum creatinine $\leqslant 1.5 \times$ of the upper limit of normal (ULN), bilirubin $\leqslant 1.25 \times$ ULN, and serum aminotransferases $\leqslant 2.5 \times$ ULN). Patients were excluded if they had other active malignancies, brain metastasis, or severe comorbid conditions. After IRB approval, informed consent from all patients was obtained before enrolment.

\section{Treatment schedule}

The starting dose of S-1 was twice daily at $35 \mathrm{mg} \mathrm{m}^{-2}$. S-1 was administered within $1 \mathrm{~h}$ after meals for 2 weeks, followed by a 1 -week rest. The schedule was repeated until the occurrence of disease progression, unacceptable toxicities, or patient's refusal. A dose reduction of $10 \mathrm{mg} \mathrm{m}^{-2}$ a day was made if $\geqslant$ grade 3 haematological or non-haematological toxicity was shown in the previous cycle. Dose re-escalation was not allowed. Patients who required more than 4 weeks of rest for recovery from any toxicity other than alopecia, nausea, vomiting, or anaemia, or who required dose reduction of more than two steps (total $20 \mathrm{mg} \mathrm{m}^{-2}$ a day), were withdrawn from the study.

\section{Evaluation of response and adverse event}

Baseline evaluations included a complete medical history, physical and radiologic examinations, performance status, complete blood count $(\mathrm{CBC})$, and biochemistries. During treatment, patients were evaluated with a weekly CBC. Physical examination, performance status, biochemistries were re-evaluated before each subsequent cycle. Imaging studies for lesions were repeated every two cycles.

Treatment response was evaluated according to the guidelines of the Response Evaluation Criteria in Solid Tumors (RECIST) Committee. Patients were considered to be assessable for response if they had evidence of early disease progression clinically or radiologically before two cycles, or if they had received a minimum of two cycles of treatment with at least one tumour measurement. If a patient was documented as having a complete response (CR) or a partial response (PR), a confirmatory evaluation was performed after 4 weeks. Time to progression (TTP) was defined as the interval from the first day of treatment until disease progression, and overall survival (OS) was calculated from the first day of treatment to death. All the patients were evaluated for adverse events weekly. Adverse events were graded according to the NCI-CTC scale (Version 3.0).

\section{Statistics}

Time-dependent variables were estimated with a log-rank test using the Kaplan-Meier method. This study was designed using the Minimax two-stage design (Simon, 1989). Sample size was calculated with $80 \%$ power to detect an objective response rate of $15 \%(\mathrm{P} 1)$ and to rule out a response rate of $3 \%(\mathrm{P} 0)$. The first stage was determined with 18 patients, and the criterion for continued accrual was the observation of at least one tumour response. The second stage was planned to accrue a further 10 patients with two more responses. The required number of patients was determined to be 28 .

\section{RESULTS}

\section{Patient characteristics}

A total of 28 patients entered the study between July 2004 and October 2005. Baseline patient characteristics are listed in Table 1.
Twenty-six patients were evaluable for tumour response. Two patients refused treatment after the first cycle and withdrew the consent. The median age was 56 years. All but four patients had undergone prior resection of their primary tumour. Liver and lung were the most common sites of measurable lesions, and peritoneal seeding was the main site of non-measurable lesions. The average diameter of target lesion was 26 (range 10-99 mm) and the median number of measurable lesions per patient was three (range 1-8).

Table 2 summarises the prior chemotherapy our patients received. S-1 was administered as third-line therapy to 21 patients and the remaining seven patients received $S-1$ as fourth-line therapy. The median time from documentation of disease progression (or relapse) of previous regimen to S-1 treatment was 21 (range 10-84) days. The median cycle number of previous chemotherapy was 18 (range 7-33) per patient, and that of regimens containing irinotecan or oxaliplatin was 6 , with a median dose intensity of 0.98 .

\section{Treatment outcomes}

A total of 125 treatment cycles (median 2, range 1-11) were administered. The median daily dose administered was 120 (range 100-150) mg. Two patients were subjected to dose reduction owing to adverse events. Seven patients voluntarily delayed their subsequent cycle by 1 week without any significant toxicity. The median dose intensity of all the patients was 317 (range 205-327) $\mathrm{mg} \mathrm{m}^{-2}$ week $^{-1}$. Seventeen patients $(60.7 \%)$ were switched over to the next chemotherapy regimens after disease progression was documented.

\section{Efficacy}

Of the 26 patients evaluable for response, one CR and three PRs were achieved, and eight patients had stable disease. The overall

Table I Patient characteristics

\begin{tabular}{lc}
\hline Patient characteristics & Number of patients (\%) \\
\hline Number of enrolled patients & 28 \\
Number of evaluable patients & 26 \\
Median age (years) (range) & $132-69)$ \\
Male: female & $15: 13$ \\
ECOG performance status & \\
$0-1$ & $8(28.6)$ \\
2 & $20(71.4)$ \\
Primary site & \\
Colon & $18(64.3)$ \\
Rectum & $10(35.7)$ \\
Histological differentiation & \\
Well moderate & \\
Poor & $25(89.3)$ \\
Prior resection of primary site & $3(10.7)$ \\
Yes & \\
No & $23(82.1)$ \\
Site of measurable lesions & $5(17.9)$ \\
Liver & \\
Lung & \\
Lymph node & \\
Abdominal mass & $49(52.1)$ \\
Metastatic site per patient & $30(31.9)$ \\
I & $12(12.8)$ \\
2 & $3(3.2)$ \\
$\geqslant 3$ & \\
\hline
\end{tabular}

ECOG $=$ Eastern Cooperative Oncology Group. 
Table 2 Summary of prior chemotherapy

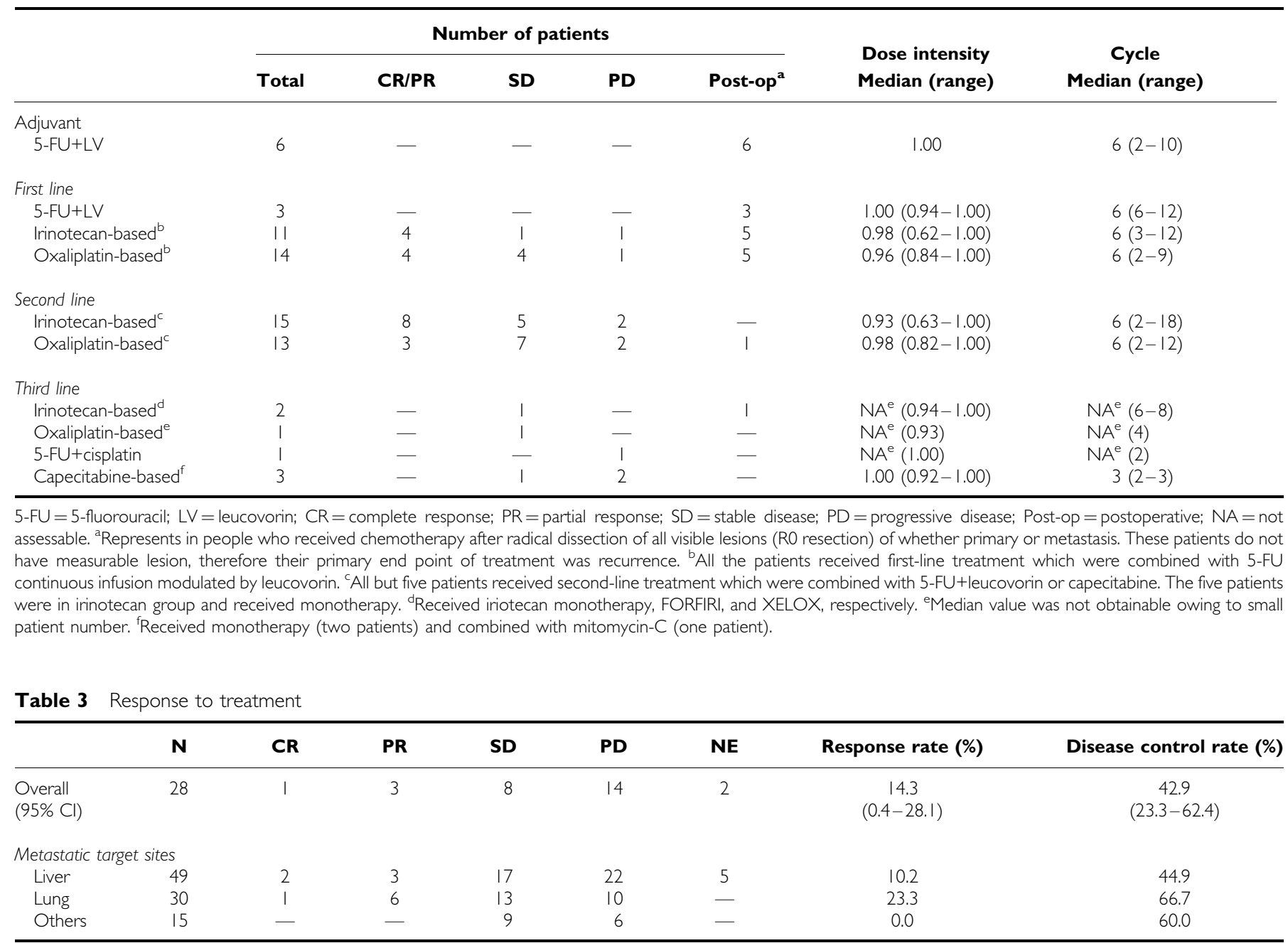

$\mathrm{N}=$ number; $\mathrm{CR}=$ complete response; $\mathrm{PR}=$ partial response; $\mathrm{SD}=$ stable disease; $\mathrm{PD}=$ progressive disease; $\mathrm{NE}=$ not evaluable; $\mathrm{Cl}=$ confidence interval; $95 \% \mathrm{Cl}, 95 \% \mathrm{Cl}$.

response rate was $14.3 \%(95 \% \mathrm{CI}, 0.4-28.1)$ and disease control rate was $42.9 \%(95 \% \mathrm{CI}, 23.3-62.4)$ by intent-to-treat analysis (Table 3). All objective responses were documented after two cycles of treatment and lasted 126, 195, 248, and 204+ days, respectively. Metastatic sites in the liver and lung had a response rate of 10.2 and $23.3 \%$, respectively, whereas other sites showed no objective tumour shrinkage. No difference in efficacy was observed with respect to the size or the number of lesions.

\section{Survival}

With a median follow-up duration of 227 (range, 28-567) days, 24 patients $(86 \%)$ showed disease progression and nine patients (32\%) expired from disease. Median TTP was 91 (95\% CI, 9-173) days (Figure 1). Median OS for all patients was 414 (95\% CI, 336492 ) days, and the 1 -year survival rate was $60.7 \%$.

\section{Safety}

No grade 4 adverse event or treatment-related mortality was observed. The most common haematologic adverse event was anaemia without evidence of bleeding, which occurred in 14 patients $(50 \%)$, with two patients receiving transfusions. Severe neutropenia (grade $\geqslant 3$ ) was reported in two patients. The most common non-haematologic adverse event was nausea, which

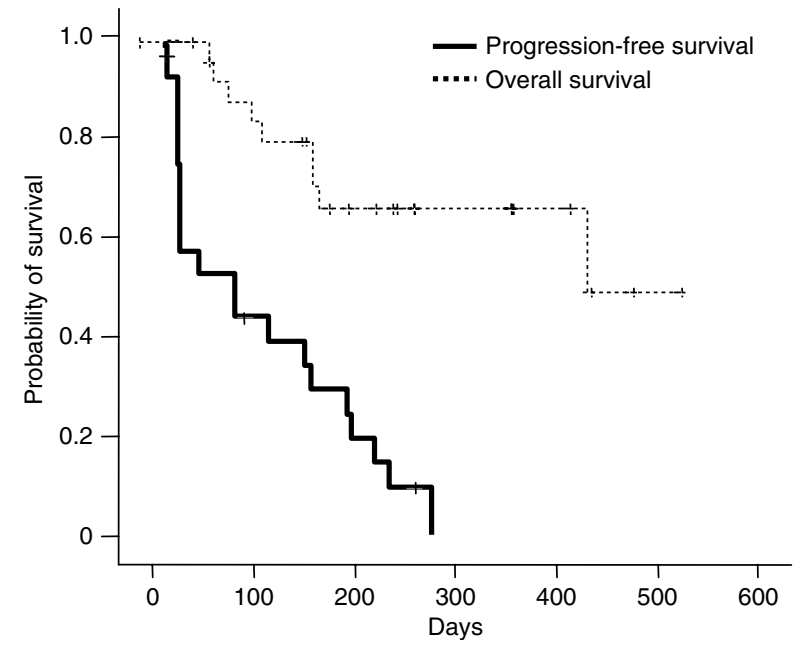

Figure I Survival analysis of all patients $(n=28)$.

affected $64 \%$ of patients. Adverse events were well managed and self-limiting. Grade 3 adverse event amenable to dose reduction was recorded in two patients, consisting of neutropenia and 


\begin{tabular}{|c|c|c|c|c|c|}
\hline & \multicolumn{3}{|c|}{ Number of patients $(\mathbf{N}=\mathbf{2 8})$} & Toxicity of all grades (\%) & Toxicity of grade 3 (\%) \\
\hline Anaemia & 7 & 6 & 1 & 50.0 & 3.6 \\
\hline Leucopenia & 7 & 4 & - & 39.3 & - \\
\hline Neutropenia & 4 & 3 & 2 & 32.1 & 7.1 \\
\hline Thrombocytopenia & 5 & - & - & 17.9 & - \\
\hline Diarrhoea & 8 & 2 & - & 35.7 & - \\
\hline Nausea & 16 & 2 & - & 64.3 & - \\
\hline Vomiting & 9 & 1 & - & 35.7 & - \\
\hline Mucositis & 9 & 2 & - & 39.3 & - \\
\hline Anorexia & 16 & I & - & 60.7 & - \\
\hline Fatigue & 7 & 1 & - & 28.6 & - \\
\hline Weight loss & 5 & 3 & - & 28.6 & - \\
\hline Abdominal pain & 10 & 1 & - & 39.3 & - \\
\hline Elevated creatinine & 2 & - & - & 7.1 & - \\
\hline Changes in liver function & 13 & 4 & - & 60.7 & - \\
\hline
\end{tabular}

anaemia combined with neutropenia, respectively. Adverse events associated with treatment are listed in Table 4.

\section{DISCUSSION}

Regimens including 5-FU with irinotecan, oxaliplatin, and bevacizumab have proven effective in the treatment of colorectal cancer as first-line and second-line treatment. However, there have been few reports of satisfactory salvage therapy in heavily pretreated patients. Novel target agents such as cetuximab have been promising for their potential role as salvage therapy (Cunningham et al, 2004). However, oral agents could be a promising alternative when taking the patients' general condition and quality of life into consideration.

Capecitabine has recently been tried as salvage therapy, but the efficacy was not satisfactory. Capecitabine showed no objective response in 5-FU-resistant cancer in a phase II trial (Hoff et al, 2004). Combination with MMC resulted in a response rate of $15 \%$ in irinotecan-resistant cancer, but its efficacy fell to $5 \%$ when the cancer was resistant to both irinotecan and oxaliplatin. Other combinations with trimetrexate or irinotecan did not show any additional benefit either (Chong et al, 2005; Gubanski et al, 2005; Matin et al, 2005).

Integrating S-1 in colorectal cancer has been proposed in several phase II studies. These studies reported a response of $24-40 \%$ with a median TTP of 5-6 months when used as a first-line treatment (Ohtsu et al, 2000; Van den Brande et al, 2003; Shirao et al, 2004). Our study is the first one investigating the role of S-1 as a salvage treatment in irinotecan- and oxaliplatin-pretreated colorectal cancer. One of our concerns in designing this phase II trial was the predictive efficacy of S-1. Our study was designed to show a response rate of $15 \%$, which is quite high considering the activity of infusional 5-FU and capecitabine in chemotherapy exposed patients. Nevertheless, this response rate is currently the minimum response expected from any new agent targeting second- or thirdline treatment for colorectal cancer (Hoff et al, 2004; Gubanski et al, 2005). Treatment schedule of S-1 was another point of consideration. Japanese trials traditionally adopted $80 \mathrm{mg} \mathrm{m}^{-2}$, but their actual dose is inconsistent with BSA $\left(64-80 \mathrm{mg} \mathrm{m}^{-2}\right.$ ) (Ohtsu et al, 2000; Shirao et al, 2004). Meanwhile, Western studies are BSA consistent. However, in a sole European phase II trial, a dose of $80 \mathrm{mg} \mathrm{m}^{-2}$ had to be reduced owing to significant non-haematological toxicity. We adopted $70 \mathrm{mg} \mathrm{m}^{-2}$ and a 3-week schedule for the purpose of procuring adequate dose intensity and compliance even in heavily exposed patients to chemotherapy. With this dosage system, we could attain high-dose intensity of $317 \mathrm{mg} \mathrm{m}^{-2}$ week $^{-1}$ (97\%).

Preclinical modelling gave several evidence that S-1 would be effective even in pretreated colorectal cancer. S-1 showed higher tumour growth inhibition than UFT did in an orthotopic implantation model of colon cancer, and S-1 promoted antitumour activity in chemoresistant cancer cells in vitro (Shirasaka et al, 1996). In a phase I study, S-1 resulted in objective antitumour activity in heavily-pretreated colorectal carcinoma, and there was a report that S-1 led to a CR in 5-FU-resistant gastric cancer (Chu et al, 1994; Tsukioka et al, 2001). All of these findings were the basis of our designing phase II trial, and we observed a response rate of near $15 \%$, which imposes a promise of S-1 for heavily pretreated patients. It is difficult to clarify the mechanisms behind the effectiveness of S-1, which are different from those of other 5FU modulators. Higher plasma or intratumoural concentrations of 5 -FU could be obtained from inhibition of dihydropyrimidine dehydrogenase by CDHP compared to simple 5-FU infusion. The inhibitory effect of CDHP on the production of $\alpha$-fluoro- $\beta$-alanine might be another explanation, as it was reported to reduce the antitumour effect of 5-FU (Cao et al, 2000).

From the perspective of survival, four patients who obtained objective tumour response had a median response duration of 200 days, suggesting that antitumour activity of S-1 was durable even in heavily treated patients once response was induced. The median TTP was 91 days, which is comparable to previously reported third-line therapy. It is surprising that OS was more than 400 days. This result might come from patient selection mechanisms irrespective of therapeutic effects of S-1. But, S-1 treatment showed favourable safety profiles even in heavily pretreated setting, resulting in median dose intensity of $97 \%$ and over $60 \%$ of patients were switched to yet other salvage regimens including 
cetuximab, capecitabine, or other novel investigational agents after failure of S-1. This suggests that patients could maintain their performance status during S-1 treatment enough to tolerate next regimen and that S-1 rarely affected the patients' compliance and quality of life. It is noticeable that the group previously exposed to capecitabine had a tendency of shorter TTP compared to the capecitabine-naïve group ( 38 days $v s 111$ days, $P=0.33$ ). Although not statistically significant owing to the small number of patients, this result implies that resistance to capecitabine may adversely influence the efficacy of S-1, and it should be an eligibility consideration for future clinical trial design.

In conclusion, this first phase II study of S-1 in irinotecan- and oxaliplatin-pretreated colorectal carcinoma demonstrated highdose intensity, promising efficacy and survival with favourable safety profile. S-1 shows potential activity as a salvage therapy in

\section{REFERENCES}

Alberts SR, Sinicrope FA, Grothey A (2005) N0147: a randomized phase III trial of oxaliplatin plus 5-fluorouracil/leucovorin with or without cetuximab after curative resection of stage III colon cancer. Clin Colorectal Cancer 5: $211-213$

Cao S, Baccanari DP, Rustum YM, Davis ST, Tansik RL, Porter DJT, Spector T (2000) alpha-fluoro-beta-alanine: effects on the antitumor activity and toxicity of 5-fluorouracil. Biochem Pharmacol 59: $953-960$

Chong G, Dickson JLB, Cunningham D, Norman AR, Rao S, Hill ME, Price TJ, Oates J, Tebbutt N (2005) Capecitabine and mitomycin C as third-line therapy for patients with metastatic colorectal cancer resistant to fluorouracil and irinotecan. $\mathrm{Br} J$ Cancer 93: 510-514

Chu QS, Hammond LA, Schwarz G, Ochoa L, Rha SY, Denis L, Molpus K, Roedig B, Letrent SP, Damle B, DeCillis AP, Rowinsky EK (2004) Phase I and pharmacokinetic study of the oral fluoropyrimidine S-1 on a oncedaily-for-28-day schedule on patients with advanced malignancies. Clin Cancer Res 10: 4913-4921

Cunningham D, Humblet Y, Siena S, Khayat D, Bleiberg H, Santoro A, Bets D, Mueser M, Harstrick A, Verslype C, Chau I, Van Cutsem E (2004) Cetuximab monotherapy and cetuximab plus irinotecan in irinotecanrefractory metastatic colorectal cancer. $N$ Engl J Med 351: $337-345$

De Gramont A, Figer A, Seymour M, Homerin M, Hmissi A, Cassidy J, Boni C, Cortes-Funes H, Cervantes A, Freyer C, Papamichael D, Le Bali N, Louvet C, Hendler D, de Braud F, Wilson C, Morvan F, Bonetti A (2000) Leucovorin and fluorouracil with or without oxaliplatin as first-line treatment in advanced colorectal cancer. J Clin Oncol 18: $2938-2947$

Douillard JY, Cunningham D, Roth AD, Navarro M, James RD, Karasek P, Jandik P, Iveson T, Carmichael J, Alakl M, Gruia G, Awad L, Rougier P (2000) Irinotecan combined with fluorouracil compared with fluorouracil alone as first-line treatment for metastatic colorectal cancer: a multicentre randomized trial. Lancet 355: $1041-1047$

Glimelius B, Hoffman K, Graf W, Pahlman L, Sjoden PO (1994) Quality of life during chemotherapy in patients with symptomatic advanced colorectal cancer: The Nordic Gastrointestinal Tumor Adjuvant Therapy Group. Cancer 73: $556-562$

Gubanski M, Naucler G, Almerud A, Lidestahl A, Lind PARM (2005) Capecitabine as third line therapy in patients with advanced colorectal cancer. Acta Oncologica 44: 236-239

Hoff PM, Pazdur R, Lassere Y, Carter S, Samid D, Polito D, Abbruzzese JL (2004) Phase II study of capecitabine in patients with fluorouracilresistant metastatic colorectal carcinoma. J Clin Oncol 22: 2078-2083 heavily pretreated colorectal cancer. Dose of $35 \mathrm{mg} \mathrm{m}^{-2}$ twice daily is feasible and can be used in designing further combination chemotherapy.

\section{ACKNOWLEDGEMENTS}

This study was supported by the Korea Science and Engineering Foundation (KOSEF) through the Cancer Metastasis Research Center at Yonsei University, and also by a grant of Korea Health 21 R\&D Project, Ministry of Health and Welfare, Republic of Korea (0412-CR01-0704-0001). We are grateful to Seung Ah Kang, Clinical Research Coordinator for her sincere help in clinical trial management and data documentation.

Kim NK, Park JK, Lee KY, Yun SH, Sohn SK, Min JS (2002) Prognostic factors influencing the recurrence pattern and survival rates in curatively resected colorectal cancer. J Korean Surg Soc 62: $421-429$

Matin K, Jacobs SA, Richards T, Wong MK, Earle M, Evans T, Troetschel M, Ferri W, Friedland D, Pinkerton R, Volkin R, Wieand S, Ramathan RK (2005) A phase I/II study of trimetrexate and capecitabine in patients with advanced refractory colorectal cancer. Am J Clin Oncol 28: $439-444$ Ohtsu A, Baba H, Sakata Y, Mitachi Y, Horikoshi N, Sugimachi K, Taguchi T (2000) Phase II study of S-1, a novel fluoropyrimidine derivative, in patients with metastatic colorectal carcinoma. Br J Cancer 83: $141-145$

Saltz LB, Cox JV, Blanke C, Rosen LS, Fehrenbacher L, Moore MJ, Maroun JA, Ackland SP, Locker PK, Pirotta N, Elfring GL, Miller LL (2000) Irinotecan plus fluorouracil and leucovorin for metastatic colorectal cancer. $N$ Engl J Med 343: $905-914$

Saltz LB, Meropol NJ, Loehrer Sr PJ, Needle MN, Kopit J, Mayer RJ (2004) Phase II trial of cetuximab in patients with refractory colorectal cancer that expresses the epidermal growth factor receptor. J Clin Oncol 22: $1201-1208$

Shirao K, Ohtsu A, Takada H, Mitachi Y, Hirakawa K, Horikoshi N, Okamura T, Hirata K, Saitoh S, Isomoto H, Satoh A (2004) Phase II study of oral S-1 for treatment of metastatic colorectal carcinoma. Cancer 100: $2355-2361$

Shirasaka T, Nakano K, Takechi T, Satake H, Uchida J, Fujioka A, Saito H, Okabe H, Oyama K, Takeda S, Unemi N, Fukushima M (1996) Antitumor activity of $1 \mathrm{M}$ tegafur-0.4M 5-chloro-2,4-dihydropyridine-1M potassium oxonate (S-1) against human colon carcinoma orthotopically implanted into nude rats. Cancer Res 56: 2602-2606

Simon R (1989) Optimal two-stage design for phase II clinical trials. Controlled Clin Trials 10: 1 - 10

Tsukioka Y, Matsumura Y, Hamaguchi T, Goto M, Muro K, Yamada Y, Shirao K (2001) Complete response achieved following administration of $\mathrm{S}-1$ in a patient with adrenal gland metastasis of 5-FU-resistant gastric cancer: a case report. Jpn J Clin Oncol 31: 450-453

Tyagi P (2005) Adjuvant irinotecan regimens in combination with infusional 5-fluorouracil/leucovorin fail to improve outcomes in surgically resected colorectal cancer. Clin Colorectal Cancer 5: 86-88

Van den Brande J, Schoffski P, Schellens JHM, Roth AD, Duffaud F, Weigang-Kohler K, Reinke F, Wanders J, de Boer RF, Vermorken JB, Fumoleau P (2003) EORTC Early Clinical Studies Group early phase II trial of S-1 in patients with advanced or metastatic colorectal cancer. $\mathrm{Br} J$ Cancer 88: $648-653$ 